\title{
Context: Technological Messes
}

Citing fears that doctored videos of political candidates could be used to manipulated in 2020, a California lawmaker has proposed legislation to ban the release of so-called deepfake [sic] images before an election.

\section{Gardiner [1]}

The contexts in which technology exists and functions are vastly more complex than what's typically assumed. The problems of society do not consist of bounded, well-structured exercises. All technologies operate in complex settings. As a consequence, the problems of society are not like the majority of simple exercises that constitute the backbone far too many classes in the Engineering and Physical Sciences, not to mention K-12 education in general.

The following is unfortunately all-too-typical of the kinds of exercises that are the basis of the body of education: " $X+6=11$; find $X$." It's certainly characteristic of the kinds of so-called problems with which earlier generations were presented. As such, it's not a problem in the true sense of the term. First of all, it's completely well defined - indeed, overly so - such that everyone is not only expected to accept as one of the key building blocks of formal education, but secondly, applying the accepted rules of arithmetic, to get the single right answer " $X=5$." In other words, exercises have one and only one "right answer" that everyone is expected to get. And, the procedures - algorithms - for getting the single right answers are clear-cut and unambiguous. Needless to say, a steady diet of exercises over a span of over 20 or more years makes many students extremely anxious and depressed when they have to confront real problems for which there are no simple, single, clear-cut answers. We know this from teaching many students and conducting numerous seminars over the course of our careers.

Real problems have none of the supposedly desirable characteristics of simple exercises. First of all, each stakeholder - once again, all the parties who affect and are affected by the problem-potentially sees it in very different ways. Thus, if

I. I. Mitroff and R. Storesund, Techlash, Management, Change,

Strategy and Positive Leadership, https://doi.org/10.1007/978-3-030-43279-9_4 
Sandra has an income of only $\$ 2000$ a month, how does she feed and care for herself and her two children if her rent and other living costs come to \$2500? The "answer" is not the simple arithmetic difference between $\$ 2500$ and $\$ 2000$. It's rooted far more in how one survives a precarious situation than it is an exercise in arithmetic. We wouldn't expect a social worker to see the problem in the same way that a parent, relative, spouse, or dependent child would. The point is that problem formulation and negotiation are two of the most important and crucial parts of problem solving. Before we can "solve" a problem, we first have to agree on its "definition."And in doing so, we have to ensure ourselves that we are not committing what's referred to as Type III Errors, "solving the wrong problems precisely." To minimize such errors, we have to engage multiple views of a problem and debate which ones best fit the situation. The earlier example of using cost as a proxy for health care is a prime case of "solving the wrong problem."

As a general rule, problems are parts of complex messy systems where the "basic problem" is not only to define the "fundamental nature of the problem," but its "relationship to the host of other problems to which it's connected in a myriad of ways." And, unlike simple exercises, Ethics plays a major role. Namely, why ought we attempt to solve this and only this set of problems versus others? In the case of technology, who will benefit from it? Conversely, who will be potentially harmed? And, how can we anticipate such harm and what can we do to mitigate it?

\section{Wicked Messes}

The late, distinguished social systems analyst and thinker extraordinaire Russell L. Ackoff appropriated the word "Mess" to stand for a whole system of problems that were so interconnected — indeed inseparable — such that one couldn't take any single problem out of the Mess and attempt to analyze it, let solve it, independently of all the other problems without distorting irreparably both the basic nature of the so-called individual problem and the entire Mess of which it was a part. The notion of "individual problems" is nothing more than a highly misleading and outdated figure of speech. The interconnections between problems are as critical, if not more so, than the so-called individual problems themselves. For example, one can't even begin to formulate, let alone "solve," the problem of "homelessness" independently of "urban crime," "income inequality," "mental illness," "drug addiction," "civic indifference and opposition," and the host of other equally critical known and unknown problems to which it's connected in a myriad of ways, both familiar and unfamiliar.

A major consequence is that all of the various elements that constitute a Theory of the Unthinkable are parts of The Tech Mess. They neither exist nor function solely by themselves.

Messes are complicated even further by an additional confounding factor; "Wickedness." A "Wicked Problem" is a problem that cannot even be formulated by any of the traditional disciplines or professions acting singly or in concert with 
one another. In other words, Wicked Problems are "beyond" the scope of any of the currently known disciplines or professions. They defy - indeed, strongly resistattempts to pin them down. In addition, a "solution," if it exists for one time and place is not necessarily one for all times and places. More often than not, so-called solutions are responsible for creating even worse problems.

Virtually all of the problems of modern societies are Wicked Messes. They are the epitome of unbounded, unstructured problems. Unlike exercises, Wicked Messes do not have simple, clear-cut, and stable solutions. One only "copes" with them as best one can. Yet, because of the historic influence of exercises, we persist in trying to pin them down and hence treat them as if they were exercises that had nice, neat, simple solutions.

In sharp contrast, Wicked Messes are only amendable to "approximate rules of thumb" or heuristics that allow us "to cope and manage them as best we can." For instance, a prime heuristic is "always be on the lookout for unanticipated-i.e., the most improbable-interactions among key factors." Another is "pay special attention to anything that threatens the most vulnerable stakeholders". 1

The Tech Mess not only contains all of the elements that comprise a Theory of the Unthinkable, but all of the parties that are involved with any and all technologies. In this way, the various stakeholders who are charged with trying to cope with a mess are fundamental parts of it. So are all of the previous attempts in trying to cope with it. Thus, the associated history of a mess is a basic part of it as well. In fact, anything that is related to a mess is part of it. In short, Wicked Messes are the epitome of unbounded, unstructured problems.

One of the saddest and most disappointing aspects of the whole issue is that the acknowledgment of Wicked Messes is still rare. As a result, we are seriously lacking in research into the heuristics that allow us to cope with them. We can't think of a more pressing topic for research.

\section{Concluding Remarks}

AI and Wicked Messes demonstrate the limits of traditional Philosophic thinking. Conventional accounts of Empiricism and Rationalism are unable to cope with the demands of Wicked Messes. They demand a different way of thinking. We say more about this later.

Once again, Thinking the Unthinkable, and thus, coping with Wicked Messes, is a key part of the job of the senior most officer involved with ensuring the Social Responsibility of an organization.

\footnotetext{
${ }^{1}$ See Mitroff et al. [2], for an expanded list of heuristics for wicked messes.
} 


\section{References}

1. Gardiner D (2019) 'Deepfake' videos would be outlawed by new bill. The San Francisco Chronicle, p D1

2. Mitroff II, Hill LB, Alpaslan CM (2013) Rethinking the education mess; A system's approach to education reform. Palgrave Macmillan, New York

Open Access This chapter is licensed under the terms of the Creative Commons Attribution 4.0 International License (http://creativecommons.org/licenses/by/4.0/), which permits use, sharing, adaptation, distribution and reproduction in any medium or format, as long as you give appropriate credit to the original author(s) and the source, provide a link to the Creative Commons license and indicate if changes were made.

The images or other third party material in this chapter are included in the chapter's Creative Commons license, unless indicated otherwise in a credit line to the material. If material is not included in the chapter's Creative Commons license and your intended use is not permitted by statutory regulation or exceeds the permitted use, you will need to obtain permission directly from the copyright holder.

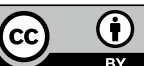

